Revue des patrimoines

$42 \mid 2020$

Imagerie numérique et patrimoine culturel :

représentation et transmission des connaissances

\title{
Donner à visiter un cuirassé dans les abysses : le « Projet Danton »
}

Letting the audience visit a battleship in the abyss: 'Danton Project'

Michel L'Hour, Daniela Peloso, Franca Cibecchini, Denis Degez, Vincent Creuze, Frédéric Osada et Christophe Leclercq

\section{(2) OpenEdition}

Journals

Édition électronique

URL : http://journals.openedition.org/insitu/28211

DOI : 10.4000/insitu.28211

ISSN : 1630-7305

Éditeur

Ministère de la Culture

Référence électronique

Michel L'Hour, Daniela Peloso, Franca Cibecchini, Denis Degez, Vincent Creuze, Frédéric Osada et Christophe Leclercq, «Donner à visiter un cuirassé dans les abysses : le « Projet Danton » », In Situ [En ligne], 42 | 2020, mis en ligne le 12 juin 2020, consulté le 12 janvier 2021. URL : http:// journals.openedition.org/insitu/28211 ; DOI : https://doi.org/10.4000/insitu.28211

Ce document a été généré automatiquement le 12 janvier 2021.

\section{(†) $\odot$

In Situ Revues des patrimoines est mis à disposition selon les termes de la licence Creative Commons Attribution - Pas d'Utilisation Commerciale - Pas de Modification 4.0 International. 


\title{
Donner à visiter un cuirassé dans les abysses : le « Projet Danton »
}

\author{
Letting the audience visit a battleship in the abyss: 'Danton Project' \\ Michel L'Hour, Daniela Peloso, Franca Cibecchini, Denis Degez, Vincent \\ Creuze, Frédéric Osada et Christophe Leclercq
}

\section{Les motivations d'un projet}

1 Près de 70 millions d'hommes ont pris part aux combats de la Première Guerre mondiale et l'on estime à près de 40 millions le nombre de ses victimes, dont 19 millions de tués. Au cours de ce conflit planétaire, les marines sous toutes leurs formes, militaire, de commerce, de pêche... ont joué un rôle de premier plan et payé un lourd tribut. Ainsi, on évalue aujourd'hui à près de 10000 le nombre des navires envoyés par le fond au cours des années 1914 à 1918, principalement par suite de l'évolution des technologies, et notamment du développement de la guerre sousmarine. Curieusement pourtant, si l'enfer des tranchées ou les prémices de la guerre aérienne sont souvent bien documentés, si les grands champs de bataille se sont très vite imposés comme des lieux de mémoire, on observe que les vestiges sous-marins, qui sont aussi un héritage tragique de ce conflit, ont été en revanche largement délaissés, peu ou prou abandonnés à la colère de la mer, voire à la rapacité de plongeurs indélicats exclusivement préoccupés d'alimenter de leur butin le commerce clandestin des « souvenirs » militaires. Cédant le pas aux « poilus » et aux collines de l'Argonne, la guerre sur mer est ainsi demeurée longtemps dans l'ombre et les campagnes du Jutland ou la première "bataille de l'Atlantique » ne sont guère évoquées qu'en termes très généraux de stratégie navale, d'évolution des technologies ou de puissance de feu. Civils ou militaires, marins ou passagers, les hommes disparus en mer pendant la Première Guerre mondiale, à l'exception notable des victimes du Lusitania, ne sont de ce fait que rarement évoqués et peu d'entre eux s'émancipent ponctuellement de l'anonymat des statistiques alors même que l'histoire de leur disparition témoigne souvent d'un héroïsme quotidien qui ne mérite pas tant de négligence. 
Conscients de cette lacune, les archéologues sous-marins du Département des recherches archéologiques subaquatiques et sous-marines (Drassm) ont décidé en 2013 d'aider le public à mieux appréhender cette page de notre histoire engloutie. Prospecter quelques-unes des épaves de la Première Guerre mondiale, étudier leur potentiel d'information, relater les circonstances de leur perte, rendre compte d'une vie à bord brutalement figée ont paru à cet égard un moyen efficace pour tenter de combler le vide qui s'était depuis un siècle sournoisement installé entre ce monde immergé et celui des vivants. De ce constat est né un programme techniquement très ambitieux : le « Projet Danton».

\section{Le Danton : un cuirassé au destin tragique}

3 Premier d'une classe de six cuirassés en acier ${ }^{1}$ dont il est l'éponyme, le Danton fut mis en chantier en 1906 à l'arsenal de Brest. Lancé en 1909 dans des conditions curieusement dramatiques ${ }^{2}$, admis au service en 1911, ce navire de $146 \mathrm{~m}$ de long pour une largeur de $26 \mathrm{~m}$ au maître-couple jaugeait 18300 tonnes et était armé de 2 tourelles doubles de 305 placées dans l'axe, à la proue et à la poupe, et de six tourelles doubles de 240 symétriquement disposées à tribord et à bâbord. Seize pièces de 75, 10 de 47 et 2 tubes lance-torpilles sous-marins latéraux complétaient cet armement. Cette redoutable machine de guerre ${ }^{3}$ était enfin servie par un équipage de 946 officiers et marins auxquels s'ajoutaient, le jour de son naufrage, 155 passagers, pour l'essentiel des marins rejoignant en Méditerranée orientale leurs bâtiments d'affectation [fig. 1].

Figure 1

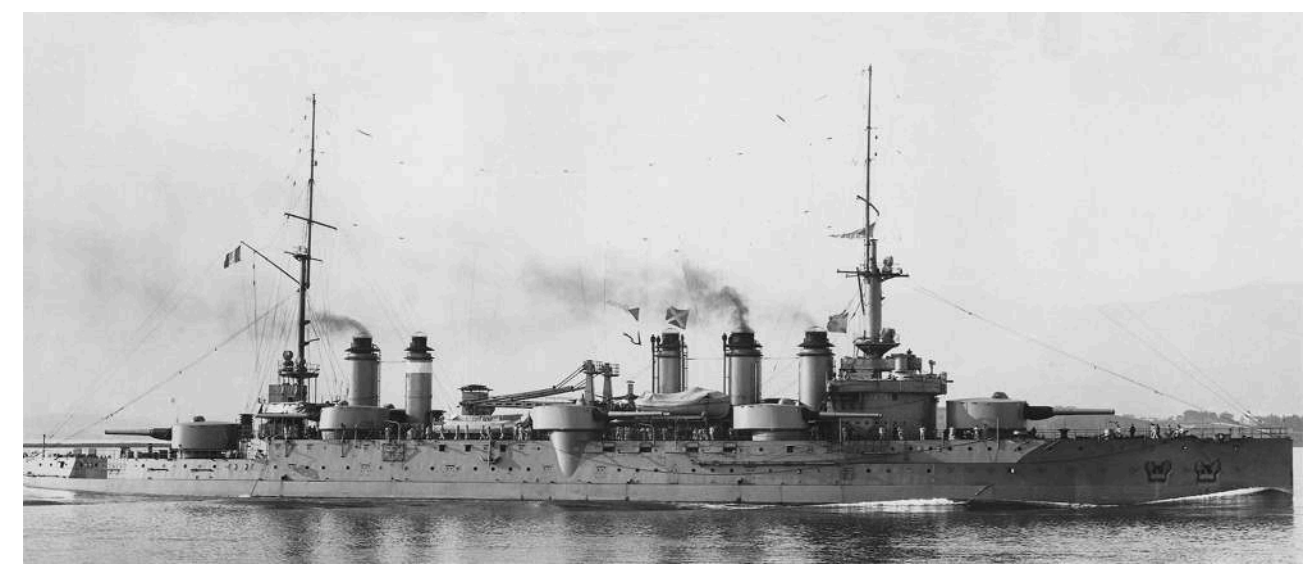

Cuirassé Danton ou de « classe Danton » en mer vers 1913.

Photographe inconnu / cliché : musée national de la Marine.

4 Escorté par le contre-torpilleur Massue, le Danton quitta Toulon le 18 mars 1917 à destination de Corfou après avoir bénéficié depuis le 13 novembre 1916 de différents travaux de modernisation. Prévenu de la présence de submersibles ennemis en mer Tyrrhénienne, son commandant, le capitaine de vaisseau Joseph Delage, alors âgé de 55 ans $^{4}$, choisit à l'appareillage de transiter par l'ouest de la Sardaigne, décision apparemment judicieuse puisque les nouvelles parvenues à l'escadre dans la matinée du 19 mars ne signalaient aucune activité sous-marine sur sa route. Nonobstant, à 13h15, le puissant bâtiment se trouvait à 28 milles dans le sud-ouest de l'île San Pietro quand la vigie de hune signala à quelques centaines de mètres sur bâbord un sillage qui 
se dirigeait droit vers le navire. Atteint, par le travers de la passerelle et en arrière de celle-ci, par deux torpilles tirées par le sous-marin allemand U-64 commandé par le Kapitänleutnant Robert Moraht ${ }^{5}$, le Danton allait couler en moins de 40 minutes, emportant dans l'abîme 296 marins, dont le commandant Delage et son état-major qui avaient refusé d'abandonner le navire. Le torpilleur Massue puis les chalutiers LouiseMarguerite et Chauveau purent sauver par chance plus de 800 marins qui s'étaient réfugiés sur des radeaux qu'on avait pu hâtivement jeter à la mer depuis le pont où ils étaient entreposés.

\section{Une prospection géophysique, une découverte inattendue, un challenge improbable}

5 Perdue à très grande profondeur très au large de Cagliari (Sardaigne, Italie), très vite oubliée, l'épave du Danton a presque miraculeusement ressurgi lorsque l'entreprise néerlandaise Fugro, qui œuvrait à des relevés géologiques sous-marins pour le compte de la société Galsi, un consortium gazier algéro-italien, a localisé en janvier 2008, par $1025 \mathrm{~m}$ de fond, une importante anomalie. Immédiatement expertisée par un robot, celle-ci a révélé l'épave en apparence bien conservée d'un grand navire d'époque contemporaine lourdement armé [fig. 2]. Très vite, l'hypothèse qu'il pouvait s'agir du Danton a été évoquée par des historiens italiens et les autorités françaises ont été alertées. Basées sur un ensemble de photos, quelques vidéos et une rapide restitution modélisée du site, les études conduites en France par le Service historique de la Défense $^{6}$ et le Drassm ont rapidement confirmé l'identité de l'épave. Il s'agissait sans doute aucun du cuirassé de Joseph Delage.

Figure 2

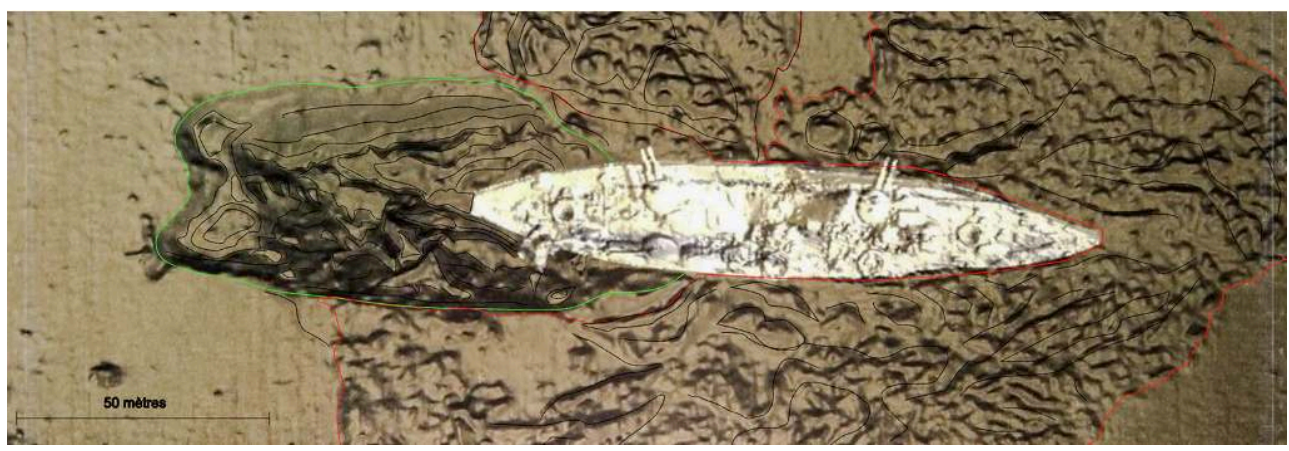

Modélisation de l'épave d'après une image sonar acquise durant la campagne de prospection effectuée par l'entreprise Fugro pour le compte de la société Galsi.

Image : Galsi.

6 À quelques années des commémorations de la Grande Guerre, cette intrusion soudaine dans l'actualité d'un cuirassé noyé depuis près d'un siècle dans l'obscurité des abysses s'est imposée d'emblée comme un signe du destin. Emblématique de la guerre navale des années 1914 à 1918, apparemment inaccessible du fait de sa bathymétrie, le Danton ne pouvait qu'être l'épave de référence que l'on donnerait virtuellement à visiter au public! Ainsi, dès 2008, le « Projet Danton " s'est lentement matérialisé. Il comportait pour les archéologues des défis innombrables puisqu'il leur restait tout à inventer: robotique dédiée, appareils de prises de vue, systèmes d'éclairage... Doté en janvier 
2012 de l'André-Malraux, un nouveau navire de recherche capable d'acheminer au large et mettre en œuvre les technologies les plus performantes, le Drassm s'est à partir de 2013 mobilisé pour mener à bien l'étude du vieux cuirassé.

7 En avril 2015 et août 2016, deux missions ont été effectuées par le Drassm à la verticale de l'épave. Dirigées par Michel L'Hour avec l'appui de Franca Cibecchini (Drassm), elles ont mis en œuvre un robot équipé de systèmes inédits de prise de vue photo et vidéo et de deux puissants éclairages spécifiquement conçus à cet effet. En moins d'une semaine, dissociée en deux campagnes sur le site, l'équipe a pu acquérir plus de 42000 photographies à haute définition des vestiges et 20 heures de vidéo. Dès 2015, ce travail a dans un premier temps permis de mieux appréhender l'épave.

Pour partie bien conservé, le Danton repose presque à plat sur un sol de vase volatile. Le tiers avant du cuirassé, de l'étrave au blockhaus de commandement, est la zone la mieux préservée. Les ancres sont à poste et des chaînes courent sur le pont cependant qu'à l'avant tribord la tourelle double est encore tournée vers le large d'où le danger peut surgir. Reposant simplement par gravité sur leur affût, les tourelles doubles de 305 ont en revanche disparu. Elles ont probablement basculé vers l'abysse lorsque le Danton a chaviré et sont peut-être à l'origine des points d'impact qu'on a pu localiser à quelque distance du site sans prendre le temps de les reconnaitre. On note sur les flancs du cuirassé la présence des bossoirs qui portaient les embarcations destinées au sauvetage. Aucune d'entre elles en revanche n'est plus en place bien qu'on sache que la bande prise par le Danton immédiatement après l'impact, associée à la panne d'électricité qui a suivi l'envahissement de la chaufferie, n'a pas permis de les mettre à l'eau. Contrastant avec la zone avant du navire, et notamment l'étrave qui demeure presque magiquement intacte, les superstructures situées en arrière de la passerelle de commandement sont littéralement broyées, laissant place à des tôles déchirées et des cavités sans fond que les robots ont renoncé à explorer. L'arrière du navire, enfin, n'est plus qu'un berceau ensablé d'où ne jaillissent que les tourelles de 240 dressées comme des phares plantés sur un îlot rocheux.

Au-delà de cette analyse détaillée des vestiges, le travail réalisé en 2015 et 2016 a surtout atteint le premier de ses objectifs en offrant l'opportunité d'une modélisation 3D de l'épave. Il a en outre conduit à improviser et à développer une technologie novatrice et performante qui permet d'envisager aujourd'hui la réalisation rapide de couvertures 3D systématiques d'épaves situées jusqu'à $2000 \mathrm{~m}$ de profondeur. Il a enfin garanti la préservation d'informations qui ne seront plus disponibles très longtemps.

10 Partout en effet, on a pu observer que la corrosion dévorait inexorablement le navire cependant que sa carène d'acier était simultanément attaquée par des bactéries mangeuses de métal, dont sans doute la fameuse Halomonas titanicae, pour la première fois mise en évidence à l'issue des opérations programmées sur le tristement célèbre Titanic de la White Star Line ${ }^{7}$. Ces bactéries attaquent aujourd'hui toutes les sections où la peinture est moins protectrice, de sorte que, colorée de jaune par les composés soufrés, la matière métallique s'écoule peu à peu en stalactites [fig. 3]. Tout l'indique en vérité, le Danton est inéluctablement condamné à disparaître et l'on peut parier que d'ici un siècle ou deux, son sort sera très certainement scellé. 
Figure 3

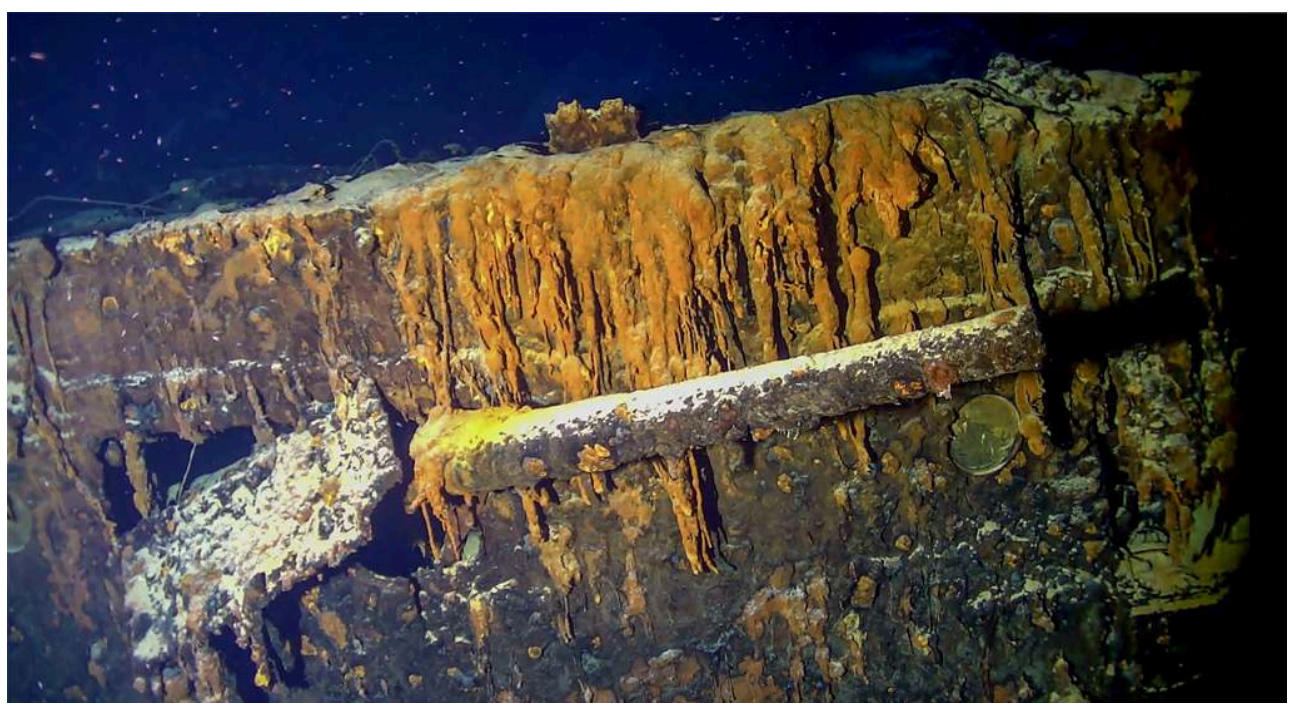

Colorées de jaune par les composés soufrés, les stalactites de métal révèlent que la carène d'acier est attaquée par des bactéries. Image capturée par le ROV.

Photo : Frédéric Osada (Images Explorations) / cliché : Drassm, ministère de la Culture.

\section{Innover pour explorer les grands fonds}

11 L'intervention sous-marine conduite à l'aide d'engins robotisés exige que l'opérateur de surface bénéficie de la perception la plus large et la plus acérée possible de l'espace où son ROV (Remotely Operated Vehicle) évolue. Si cette exigence est assez universellement admise, elle est cependant très inégalement satisfaite. Force est de constater que les angles de vue aujourd'hui offerts aux pilotes des ROV industriels et militaires sont souvent sous-dimensionnés, d'où des temps d'approche accrus afin de garantir une marge de sécurité suffisante et une bonne mémorisation spatiale du futur espace d'évolution. Si, avec l'habitude, bon nombre de pilotes s'accommodent de la situation, pour l'archéologue en revanche la vision demeure cruciale. Elle n'est même, à vrai dire, pas négociable sur les épaves, tout particulièrement sur les épaves contemporaines dont l'environnement est souvent chaotique, donc " piégeant », et, au moins en approche initiale, totalement inconnu.

À ce premier impératif qu'est la vue s'ajoute pour l'archéologue celui de s'assurer d'une production d'images d'une définition suffisante pour documenter de manière efficiente, scientifiquement et techniquement, le site investigué, voire pour satisfaire aux besoins de sa communication audio-visuelle. Tout au long de ce processus, on l'aura compris, c'est donc la qualité de l'image qui s'impose au cœur des préoccupations au point qu'elle seule peut in fine justifier la mobilisation de l'ingénierie maritime et sousmarine nécessaire à son acquisition.

13 Afin d'envisager avec quelque chance de succès la modélisation de sites localisés à des profondeurs où la lumière ne pénètre pas et où la pression se fait énorme, il est très vite apparu que l'on ne pourrait se contenter d'importer des méthodes et des systèmes préexistants. Ce constat ne signifie pas bien sûr que nul ne se soit avant nous préoccupé d'assurer l'analyse des épaves situées à grande profondeur. Souvent favorisées par une préservation exceptionnelle, grâce à une bathymétrie qui les laisse hors d'atteinte des 
prédateurs humains tout en les conservant dans un milieu bénéficiant de basses températures et d'un faible taux d'oxygène, ces épaves constituent bien au contraire un sujet de recherche scientifique privilégié pour les quelques spécialistes capables aujourd'hui dans le monde de s'attaquer au problème. Les biens culturels maritimes des abysses ont donc ponctuellement suscité au fil des années le développement de technologies plus ou moins bien adaptées à leur milieu d'enfouissement ${ }^{8}$, et maintes expériences ont été conduites pour dévoiler et relever, par un éclairage adéquat et de manière non destructrice, des sites ${ }^{9}$ très profonds (au-delà de 500 mètres). Mais si la diffusion de ces méthodes a effectivement permis de développer des dispositifs de contrôle et des capteurs optiques, il demeure que, pour répondre au cahier des charges particulièrement ambitieux énoncé par le Drassm dans le cadre du « Projet Danton », il convenait de réaliser un vrai bond technologique dans le domaine des éclairages et de la prise de vue sous-marine. Les capteurs et les « douches lumineuses » disponibles « en magasin» pour réaliser la couverture des gisements sous-marins accessibles aux plongeurs n'ont en effet pas leur équivalent pour les sites très profonds. La solution devait nécessairement passer par une phase de recherche et de développement. Elle a été confiée en 2014 à Frédéric Osada (Images Explorations / Drassm), cependant que l'épave du Danton s'érigeait en laboratoire de référence pour fonder une stratégie opérationnelle, improviser des solutions propres à pallier les carences de la technologie et tester les prototypes nés de l'ingénierie développée à cette occasion.

\section{Une technologie en devenir}

14 Le challenge n'allait pas de soi. L'épaisseur des hublots sous-marins capables de résister à la profondeur maximale d'intervention - fixée à cette occasion par le Drassm à 2000 mètres de fond, soit une pression de 200 bars (soit $200 \mathrm{~kg} / \mathrm{cm}^{2}$ ) - est susceptible d'induire des distorsions géométriques très fortes des images, ainsi que des aberrations chromatiques. De ce fait, la réalisation de lentilles appropriées, combinant l'aptitude aux pressions extrêmes et les corrections optiques rendues nécessaires par leur épaisseur, transforme de simples hublots en appareils de haute technologie particulièrement difficiles à mettre au point. La question de l'éclairage indispensable à la réalisation des images n'est pas anecdotique non plus, dès lors qu'il s'agit d'illuminer, sans zone d'ombre ni point chaud et sans apport d'éclairage naturel, un site éloigné de quatre à quinze mètres de l'objectif.

Pas moins de six partenaires et prestataires, scientifiques, laboratoires de recherche et industriels, ont été sollicités au cours de ce processus et ont accepté de mettre leur savoir-faire en commun pour concevoir et réaliser les systèmes d'éclairage et de prise de vue indispensables ${ }^{10}$. Il s'agit de :

- la société Becom-d (Frédéric De San Nicolas) qui a assuré la conception et le montage des caissons photo et vidéo ;

-Vincent Creuze, enseignant-chercheur au Laboratoire d'informatique, de robotique et de microélectronique de Montpellier (LIRMM) et directeur de la « robotique » du Drassm, qui a pris en charge, en partenariat avec Antoine Lefaucheux de la société Deep Vision, l'électronique de commande de la vidéo puis a réalisé avec Jérôme Sialelli (société Copetech$\mathrm{SM})$ l'ensemble de la connectique reliant les éclairages et les caissons de prise de vue au robot $P$ erseo ${ }^{11}$ sélectionné pour être le véhicule-support des équipements ; 
- l'entreprise Sophia Conseil (Guillaume Cassar) qui a réalisé l'étude et les plans des lentilles et du hublot;

- la société Galioptic, qui s'est chargée de la fabrication des hublots ;

- la société Posseïdon LDY (Christophe Leclercq) qui a conçu et réalisé les projecteurs à LED ;

- la Fondation Dassault (présidée par Marie-Pierre Aulas) qui a pour partie assuré le mécénat de l'opération Danton 2016.

Figure 4

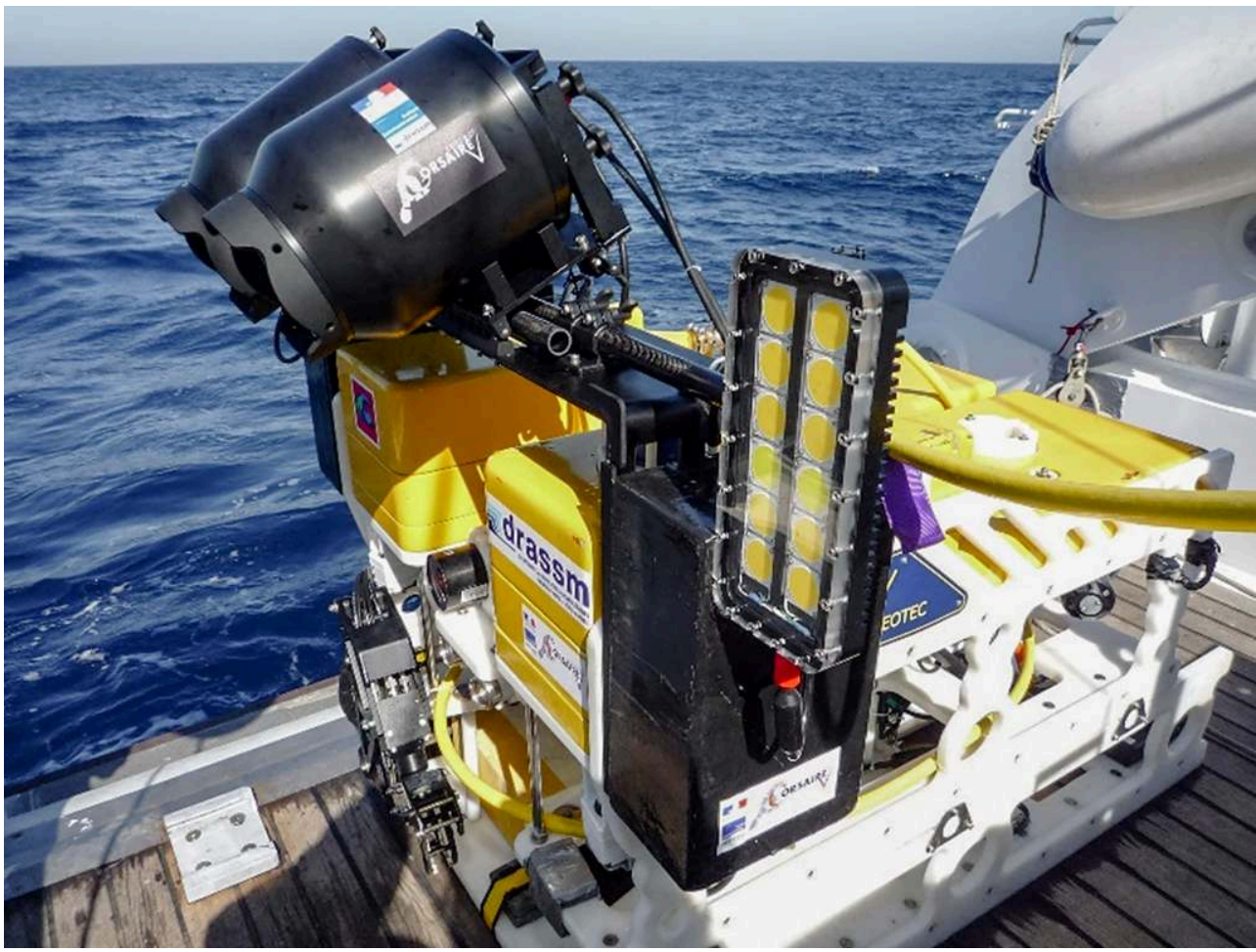

Le Remotely Operated Vehicle (ROV) Perseo GTV intègre deux caissons, caméra et appareil photo, placés sur le dessus du ROV, et deux puissants projecteurs à LED (340 000 lumens chacun). Une fois dans l'eau, le système d'éclairage assure une visibilité jusqu'à 25 mètres du ROV. L'angle d'inclinaison de la caméra est ici de $30^{\circ}$.

Photo : Michel L'Hour / cliché : Drassm, ministère de la Culture.

Le matériel ainsi développé [fig. 4] se décompose comme suit :

- un appareil photo Nikon D810, conditionné en caisson, doté d'une caméra d'œilleton et équipé d'un objectif à focale fixe de $20 \mathrm{~mm}$ Nikkor AF-S $20 \mathrm{~mm} \mathrm{f/1.8G} \mathrm{ED,} \mathrm{dont} \mathrm{la} \mathrm{mise} \mathrm{au}$ point motorisée est contrôlée depuis la surface. Cet équipement autorise normalement un champ de vision de $83,8^{\circ}$. Pour tenter de maintenir ce grand angle également dans l'eau, des hublots spécifiques ont été conçus, en tâchant de minimiser les distorsions géométriques et les aberrations chromatiques ${ }^{12}$. L'alimentation électrique de l'appareil est assurée en continu par le ROV Perseo et l'ensemble est dimensionné pour permettre le stockage en une seule plongée de 6300 prises de vues de $7460 \times 4912$ pixels, simultanément aux formats brut NEF et compressé JPEG.

- Une caméra Full HD Sony EV7500 avec sortie HD-SDI inscrite dans un caisson en aluminium anodisé résistant aux pressions rencontrées à 2000 mètres de profondeur. L'angle d'inclinaison de la caméra peut être réglé manuellement par pas de $5^{\circ}$, depuis $0^{\circ}$ (horizontal) jusqu'à $90^{\circ}$ (vertical). Les vidéos, d'une résolution de 1920 x 1080 pixels, sont stockées sur disque dur SSD par un enregistreur Samouraï Blade-Atomos au format Pro-Res $422 \mathrm{HQ}$. 
Alimentée directement par le ROV, la caméra est dotée d'un zoom optique et elle dispose d'une autonomie d'enregistrement de 11 h 25 dans sa meilleure résolution.

- Les appareils de prise de vue sont pilotés à distance par un tableau de commande à microcontrôleur conçu par le LIRMM et Deep Vision. Cette interface permet à l'opérateur de surface de contrôler manuellement ou automatiquement l'appareil photographique et la caméra en temps réel. Elle permet également d'ajuster manuellement la mise au point.

- L'éclairage est fourni par deux projecteurs à LED de 280000 lumens chacun, portés à 340000 lumens lors de la campagne de 2016. Alimentés depuis la surface (6000 W), ils sont montés sur des bras qui permettent, lorsqu'ils sont déployés, de déporter l'éclairage de $1 \mathrm{~m}$, de part et d'autre du ROV, de façon à garantir un éclairage homogène du site photographié et filmé. La puissance de l'éclairage a ici un caractère crucial puisqu'elle permet à la fois d'accélérer l'acquisition photogrammétrique, d'augmenter la vitesse d'obturation et de limiter ce faisant les risques de «flou » lors des accélérations du ROV. Elle profite enfin à la profondeur de champ car elle permet de réduire l'ouverture du diaphragme.

Grâce au partenariat mis en place, l'ensemble des questions posées à la fin de l'année 2014 a été résolu en amont de la première campagne programmée sur le Danton en avril 2015 [fig. 5]. Les problèmes, logiquement inévitables, surgis au cours de cette opération ont à leur tour trouvé des solutions en 2016, de sorte que la couverture 3D de l'épave du Danton a été finalisée dès la seconde campagne sur le site, en août-septembre 2016.

Figure 5

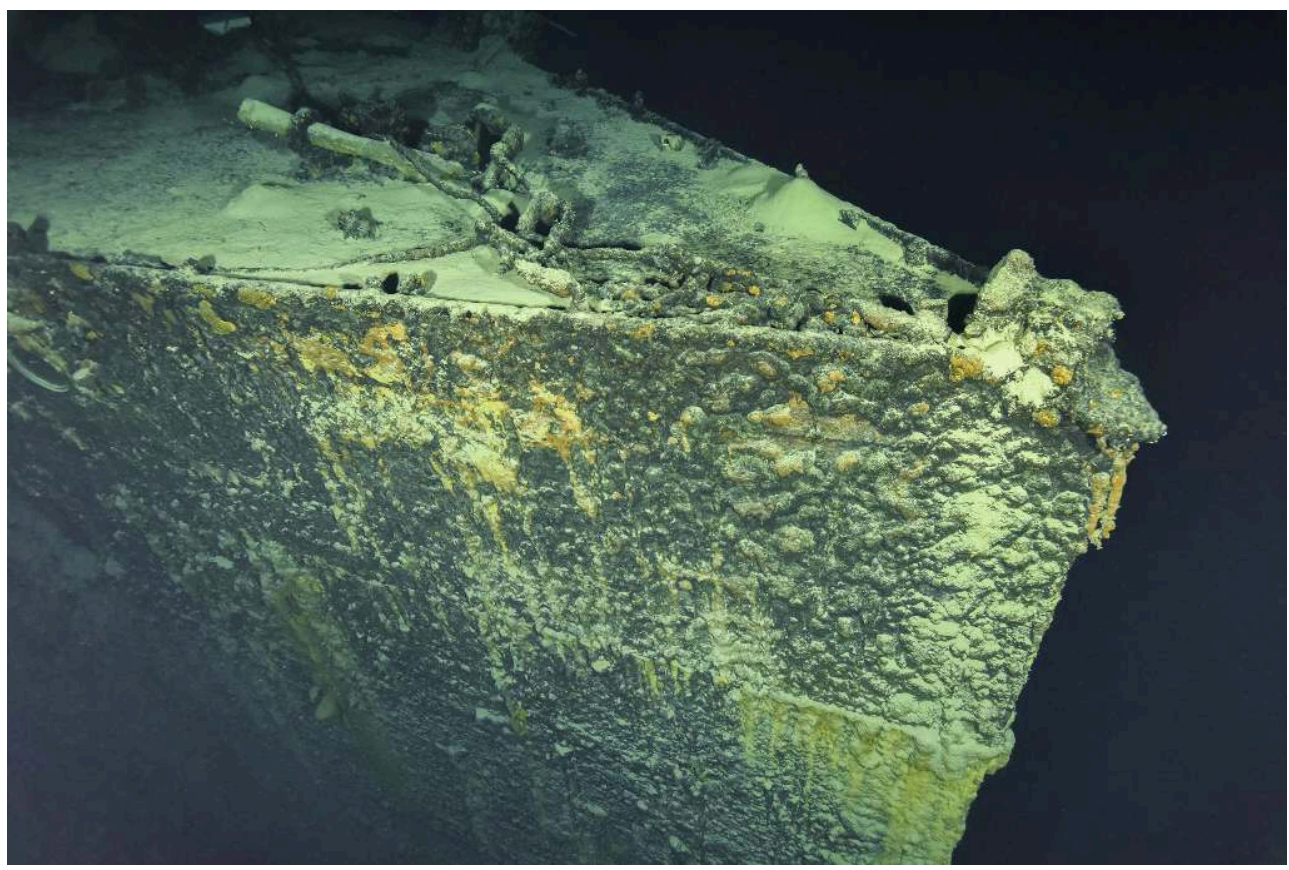

La puissance des éclairages du robot permet d'illuminer des sections conséquentes de l'épave. Ici l'étrave.

Photo : Frédéric Osada (Images Explorations) / cliché : Drassm, ministère de la Culture. 


\section{De la théorie à la pratique : vaincre au quotidien les difficultés opérationnelles}

Stationné à la verticale de l'épave, l'André-Malraux maintient sa position grâce à ses capacités de positionnement dynamique (DP) [fig. 6]. En immersion, le ROV, qui est relié au bateau par un ombilical constitué notamment de plusieurs fibres optiques, est pour sa part localisé grâce au système de positionnement acoustique sous-marin USBL (Ultra Short Baseline) de type GAPS ${ }^{13}$ installé à bord de l'André-Malraux. Basée sur des gyroscopes à fibre optique, la centrale inertielle interne (IMU $\left.{ }^{14}\right)$ du GAPS simplifie considérablement l'étalonnage du système. En opération, le transducteur USBL, ou antenne acoustique, est placé à l'extrémité d'une perche fixée sur le flanc tribord du navire, de sorte que le transducteur demeure immergé de plusieurs mètres sous la surface. Ce transducteur communique avec un transpondeur placé sur le ROV. Cet équipement permet de mesurer la distance entre le ROV et le navire avec une précision de $0,06 \%$ de la distance oblique. En combinant les mesures acoustiques avec les données de l'IMU, le transducteur détermine également l'azimut du ROV puis, à l'aide d'un récepteur GPS différentiel installé sur l'André-Malraux, calcule la position géographique du robot dans les trois dimensions (latitude, longitude, profondeur). Il est ainsi possible pour les opérateurs de suivre en temps réel la trajectoire de la machine et d'obtenir incidemment le géoréférencement de chaque image.

Figure 6

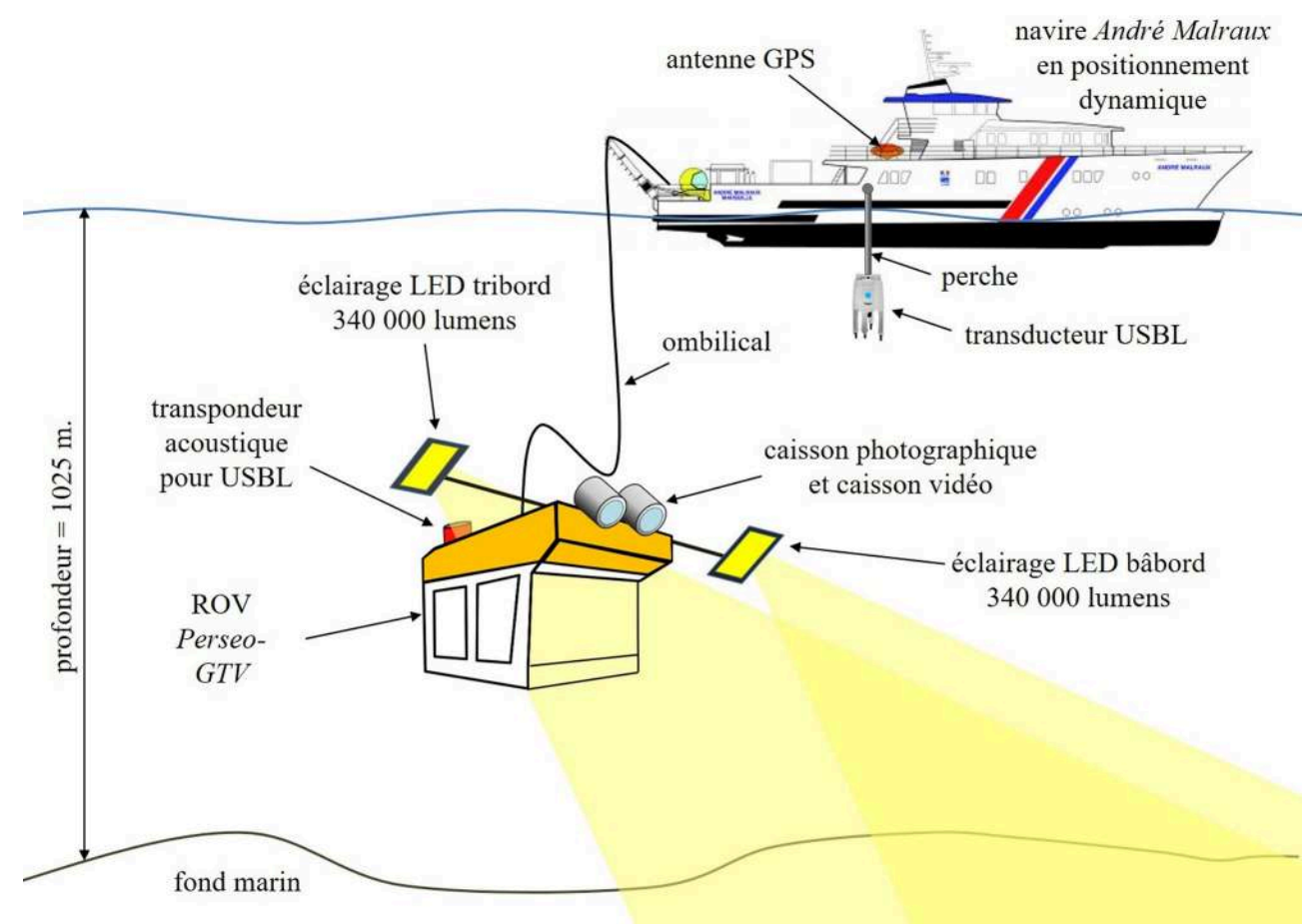

Le ROV Perseo est mis en œuvre depuis le navire de recherche André-Malraux. Ce dernier dispose de capacités de positionnement dynamique qui lui permettent de maintenir une position fixe à la verticale de l'épave.

Schéma : Drassm, ministère de la Culture.

Pour assurer le relevé complet du Danton, l'épave a été sectorisée en six zones de travail successives, de l'étrave à la poupe et sur les deux flancs, sur la base des photos réalisées 
en 2015 et du plan de construction du cuirassé retrouvé dans les archives. Les prises de vue, tant verticales qu'horizontales, ont été réalisées à une distance de 2 à 4 mètres de l'épave sans jamais venir au contact. Aucun marqueur ni règle n'a été déposé au préalable sur le site.

Les connaissances acquises lors de la campagne de 2015 ont permis de programmer en amont de l'opération de 2016 un cheminement du ROV à même de garantir une couverture exhaustive de l'épave vue de dessus et depuis les flancs. Ainsi, afin de minimiser les risques liés aux contraintes du site mais aussi aux variations de position et de vitesse du ROV, les survols ont été effectués par bandes plus ou moins parallèles, avec une zone de recouvrement de $80 \%$ d'un cliché à l'autre sur la route suivie par le ROV, et de $60 \%$ avec les clichés du parcours latéral voisin. Ce même critère de précaution a conduit à privilégier une haute fréquence de déclenchement des prises de vue (1,2 secondes). L'acquisition photographique s'est déroulée sur trois jours selon le plan de travail suivant :

- Première plongée : relevé du flanc bâbord et tribord, avec des passages verticaux à raison de 54 lignes sur bâbord et de 45 lignes sur le flanc opposé. Inclinaison de la caméra à $30^{\circ}$. (Temps de plongée: 5 heures et $30 \mathrm{~min}$. Temps de travail au fond: 3 heures et $10 \mathrm{~min}$. Nombre total de photos: 3461 ).

- Deuxième plongée : réalisation de passages verticaux tout au long du flanc tribord sur 65 lignes. Le ROV a ensuite effectué des survols autour de la première tourelle avant tribord et de la passerelle. (Temps de plongée: 5 heures et $30 \mathrm{~min}$. Temps de travail au fond: 3 heures et $10 \mathrm{~min}$. Nombre total de photos : 5 751).

- Troisième plongée : couverture photo des éléments architecturaux en élévation (passerelle, tourelles, etc.), depuis l'avant du navire vers l'arrière et retour, suivant des transects transversaux sur 30 lignes. (Temps de plongée : 4 heures et $20 \mathrm{~min}$. Temps de travail au fond : 2 heures et $25 \mathrm{~min}$. Nombre total de photos : 4 500).

- Quatrième plongée : couverture photo de la partie avant tribord (tourelle) et de la passerelle puis de la zone symétrique bâbord. Deux passages parallèles sur l'axe longitudinal aller/ retour. (Temps de plongée : 6 heures et $10 \mathrm{~min}$. Temps de travail au fond: 3 heures et 50 min. Nombre total de photos : 6500 ).

- Cinquième plongée: acquisition de l'ensemble du bateau, de l'avant vers l'arrière. Réalisation d'un profil central, selon l'axe longitudinal, afin de visionner les «cratères " ouverts par les explosions des torpilles au centre du navire. (Temps de plongée : 4 heures et $50 \mathrm{~min}$. Temps de travail au fond : 2 heures et $30 \mathrm{~min}$. Nombre total de photos : 6500 ).

21 Rythmée par la nécessité de donner du temps, d'une part aux opérateurs pour se reposer, d'autre part pour décharger et sauvegarder les cartes mémoires de l'appareil photo, la mission a donc réclamé en 2016 un total de cinq plongées pour une durée totale de $25 \mathrm{~h} 04 \mathrm{~min}$, dont 14 heures et $25 \mathrm{~min}$ de travail au fond. Elle a permis d'acquérir à cette occasion 26712 photographies.

\section{Le travail de restitution}

Les images acquises au cours de la campagne 2016 ont d'abord été sélectionnées afin de conserver uniquement les clichés les mieux adaptés au processus photogrammétrique ${ }^{15}$. Sur la totalité des photographies sauvegardées, 25000 environ ont été au final mises à contribution. 
Le premier tri a porté sur les clichés, dits de test, réalisés pour vérifier le bon fonctionnement de l'appareil photo, afin d'éliminer les images qui manquaient de netteté, étaient trop sombres ou encadraient seulement le fond marin. Les images sélectionnées témoignent d'une hétérogénéité marquée, par suite d'un environnement archéologique très difficile et de l'absolue obscurité. Pour cette raison, les images ont été regroupées selon différents critères [fig. 7]:

- zones du bateau photographiées,

- conditions d'éclairage,

- transparence de l'eau,

- distance de l'objectif par rapport au corps du navire.

Figure 7
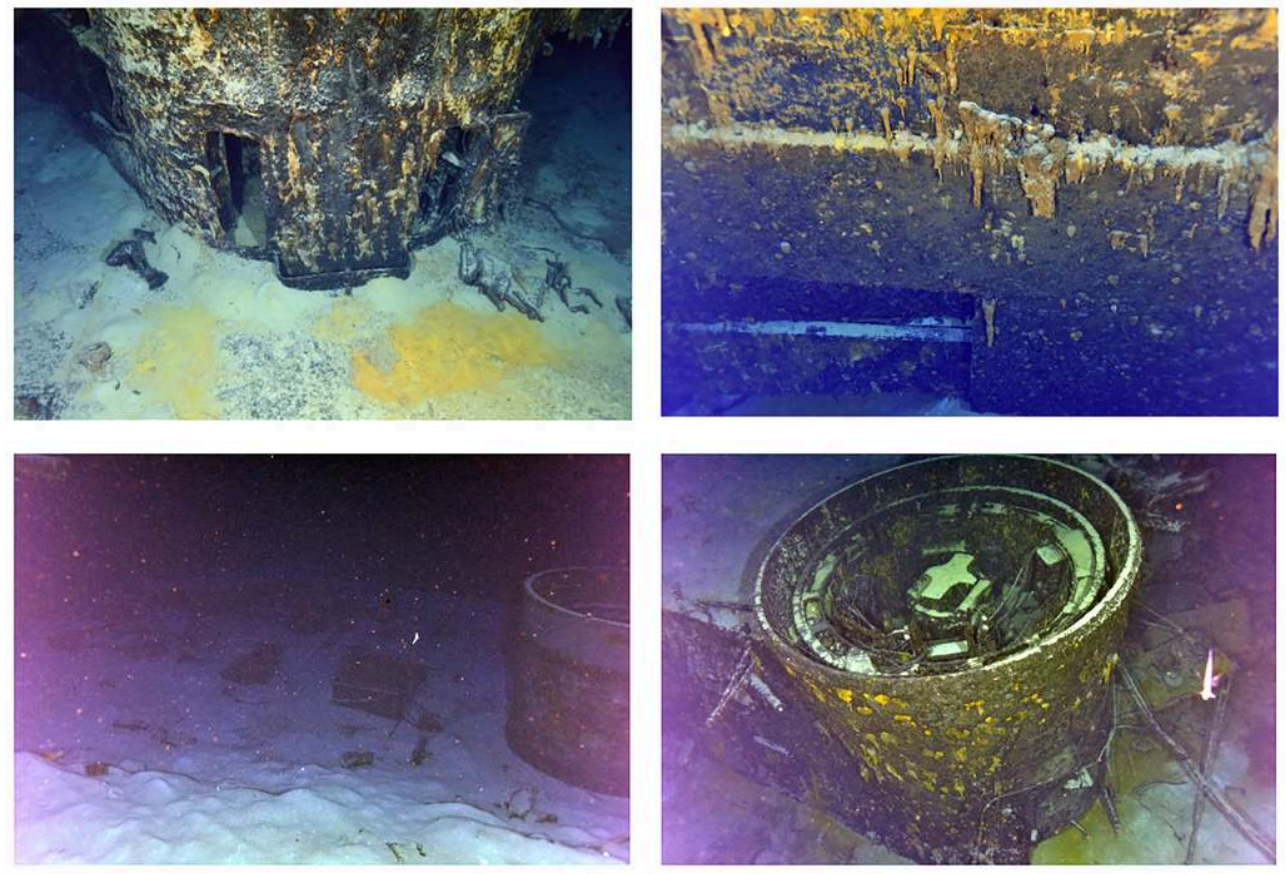

Ces photos illustrent l'hétérogénéité des prises de vue.

Photos : Frédéric Osada (Images Explorations) / cliché : Drassm, ministère de la Culture.

En milieu sous-marin, les conditions de vision se dégradent vite. Avant de lancer le processus photogrammétrique, il est donc nécessaire de soumettre les images à des traitements préliminaires dans le but d'améliorer leur qualité. Les données colorimétriques, en effet, sont soumises aux contraintes imposées par la nature du milieu: turbidité de l'eau consécutive à la présence de particules en suspension, diffusion et absorption de la lumière, etc. En pénétrant dans l'eau, l'intensité lumineuse diminue en fonction de la profondeur et l'absorption sélective génère une modification de la vision des couleurs [fig. 8]. Les radiations qui composent le spectre solaire sont progressivement absorbées dans l'ordre décroissant des longueurs d'ondes, depuis les plus élevées (infrarouge, rouge, jaune) jusqu'aux plus faibles (vert, bleu, violet, ultraviolet $)^{16}$. Ces phénomènes ne sont pas liés à la profondeur mais à la longueur de trajet du rayon lumineux dans l'eau. C'est la raison pour laquelle l'éclairage artificiel restitue leurs couleurs aux objets en dépit de la bathymétrie du site. 
Figure 8

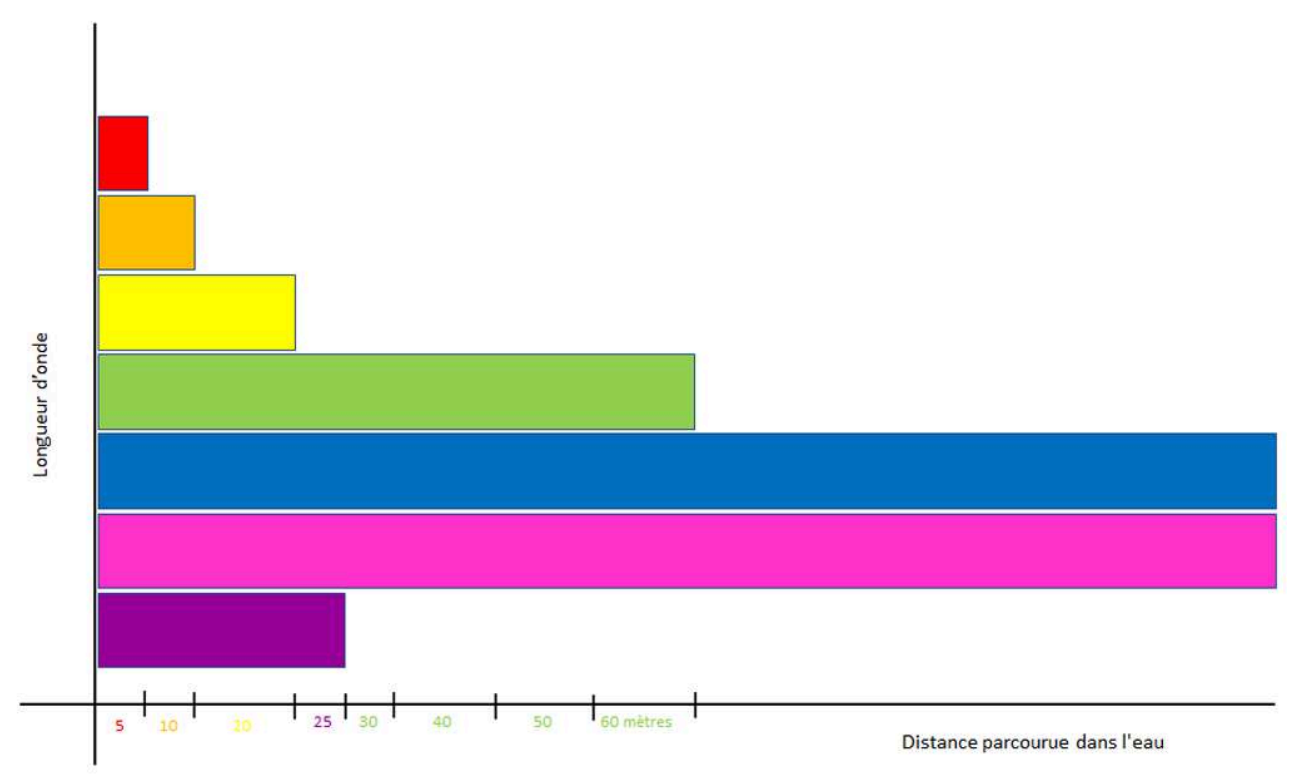

La disparition des couleurs en environnement marin.

Schéma : Daniela Peloso (Ipso Facto).

25 Les techniques dédiées à l'amélioration des images accentuent la netteté des caractéristiques de la photo ${ }^{17}$. Elles procèdent de la manipulation des niveaux de couleur, de l'égalisation d'histogramme et de l'amélioration du contraste dans le but de rendre la luminosité uniforme sur toute l'image. L'objectif est bien sûr d'augmenter la compréhension et l'interprétation des clichés [fig. 9].

Figure 9

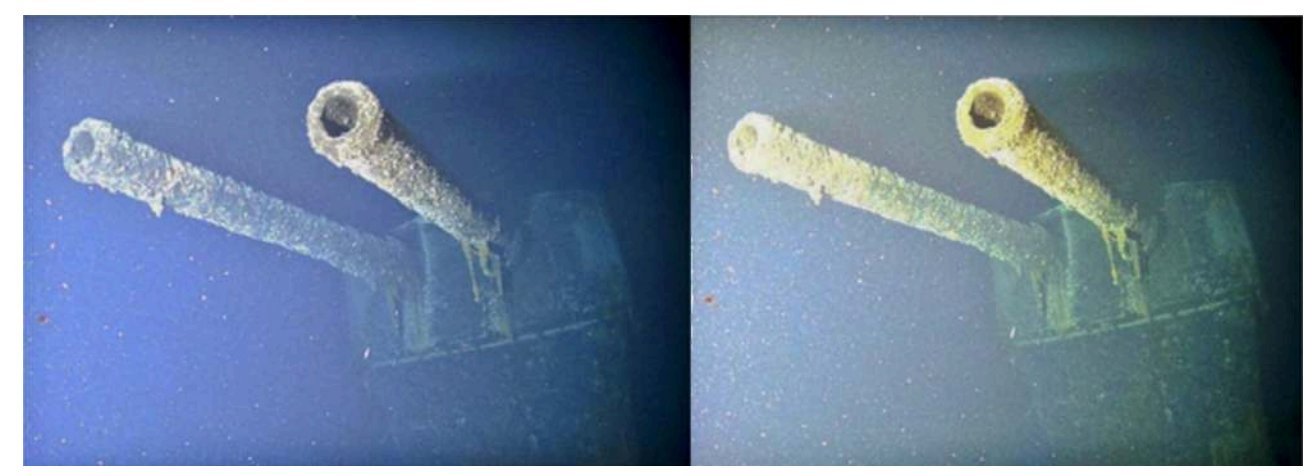

Les techniques de traitement d'image permettent de rehausser les contrastes, d'améliorer la netteté, ou encore d'équilibrer l'exposition et les couleurs. À gauche : l'image originale. À droite : l'image après traitement.

Photo : Frédéric Osada (Images Explorations) / cliché : Drassm, ministère de la Culture.

Quelquefois, il est aussi nécessaire d'intervenir pour créer sur les photos un masque afin de circonscrire l'objet à modeler et d'éliminer les zones à ne pas reconstruire. Ce procédé permet également de recadrer les photos, de façon à supprimer les déformations potentielles en périphérie, induites par l'objectif et le hublot [fig. 10]. 


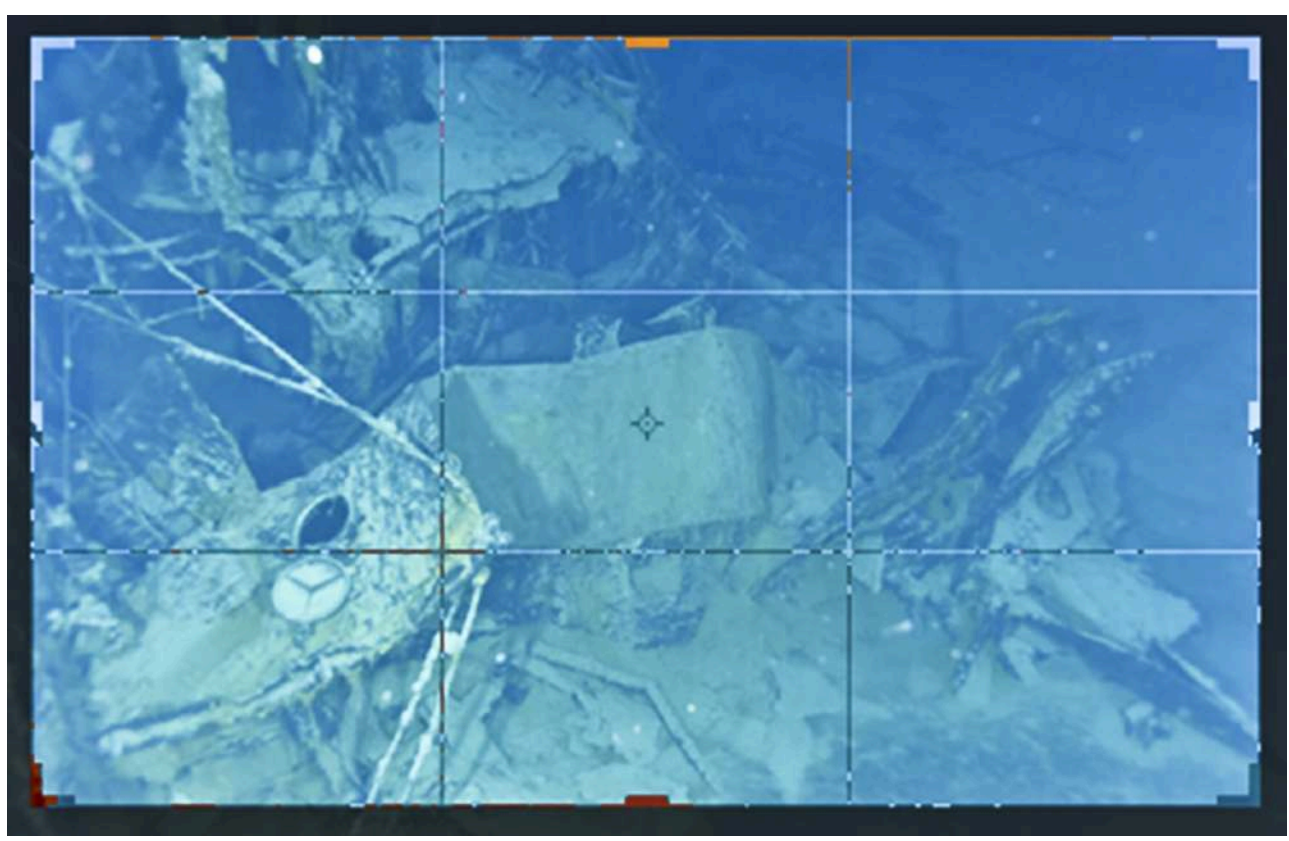

Exemple de masque pour recadrer les photos.

Photo : Frédéric Osada (Images Explorations) / cliché : Drassm, ministère de la Culture.

Les images traitées ont une définition de $300 \mathrm{dpi}$ et une taille de 102 Mo chacune. Compte tenu de ce "poids numérique " et du nombre de clichés nécessaires pour assurer la couverture d'une épave aussi imposante que celle du Danton, le temps de calcul nécessaire au processus photogrammétrique global est loin d'être négligeable.

\section{Un processus photogrammétrique chronophage mais nécessaire}

Sur l'épave du Danton, l'objectif était d'obtenir au final un modèle 3D doté d'une bonne résolution et d'une texture colorimétrique proche de celle de l'épave. L'intérêt pour les archéologues de s'assurer d'une modélisation fidèle de ce site méconnu était notamment de vérifier la possibilité d'en extraire des informations inédites sur le naufrage.

Le processus d'élaboration d'un modèle texturé découle de plusieurs niveaux successifs de calcul ${ }^{18}$. Il convient d'abord de procéder à l'alignement des photos, c'est-à-dire de calculer pour chaque vue, en fonction du sujet photographié, l'orientation et la position relative des caméras ${ }^{19}$. À cet effet, des points caractéristiques (feature points) sont identifiés sur les images afin de les apparier d'une image à l'autre pour calculer la position et l'orientation de l'appareil photo [fig. 11]. Une fois le calcul effectué par la méthode d'ajustement de faisceaux (bundle adjustment) on obtient un nuage épars de points 3D puis on génère un nuage plus dense à très haute définition en recherchant un compromis entre la précision de l'information à recueillir et la pertinence de cette dernière au regard du sujet traité [fig. 12]. 
Figure 11

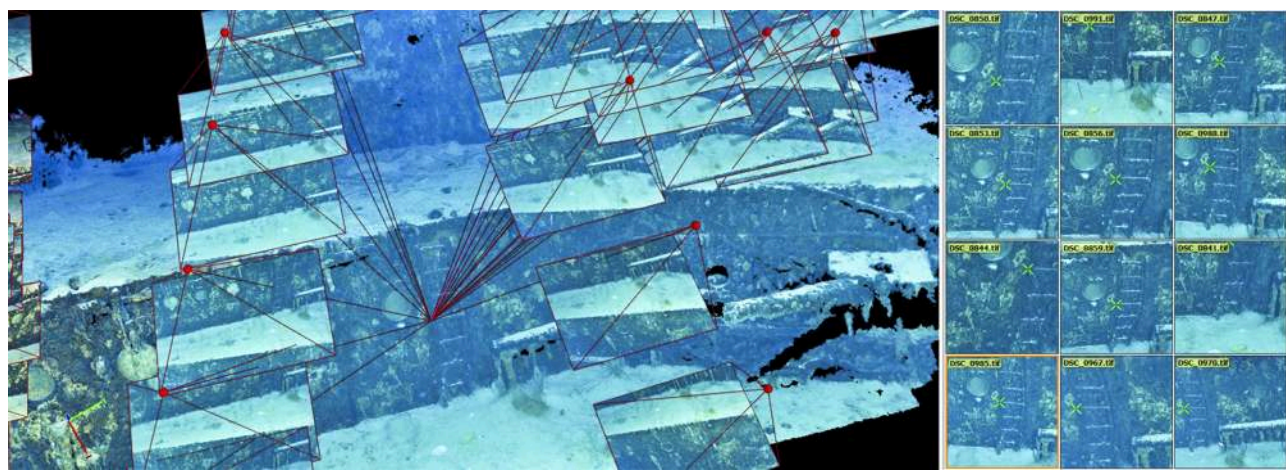

Des dizaines, voire des centaines, de points dits « points caractéristiques » sont suivis d'une image à l'autre, ce qui permet de calculer la position de l'appareil photo pour chaque photo (partie gauche de la figure). À droite, par exemple, on observe le suivi d'un même point caractéristique (bord d'une échelle) dans 12 images différentes.

Traitement : Daniela Peloso (Ipso Facto) / cliché : Drassm, ministère de la Culture.

Figure 12
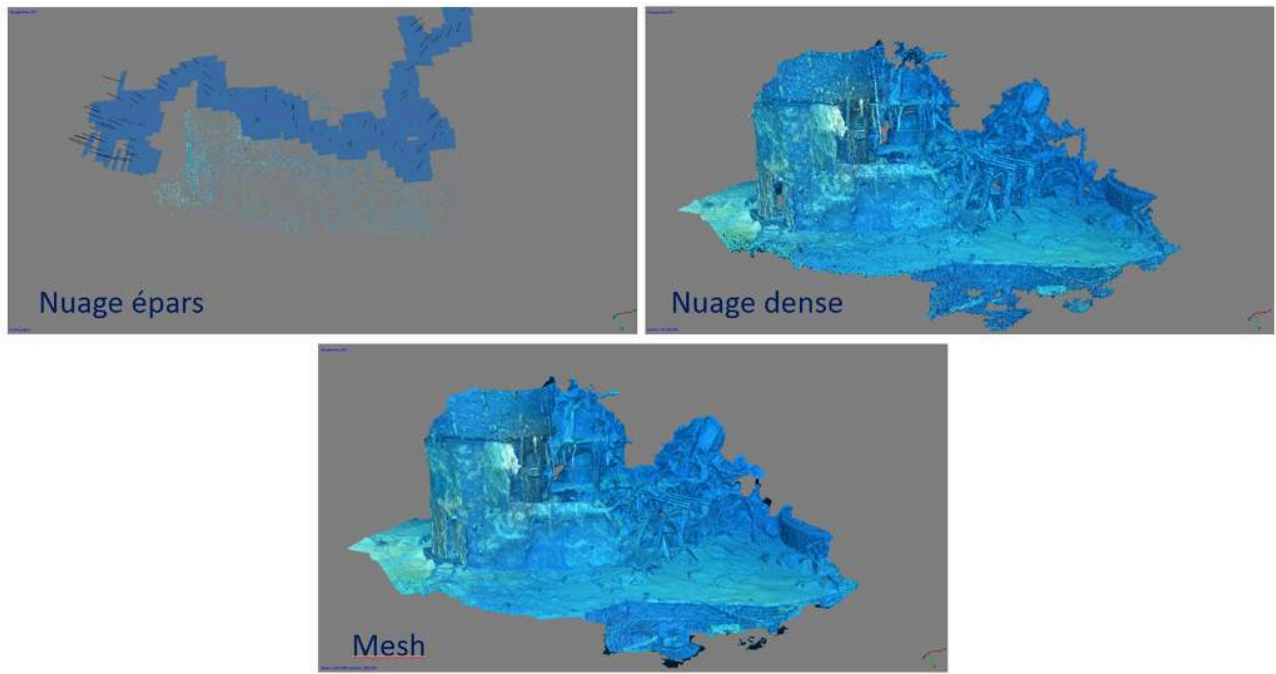

Différentes étapes de la modélisation 3D par photogrammétrie.

Traitement : Daniela Peloso (Ipso Facto) / cliché : Drassm, ministère de la Culture.

L'étape suivante de la chaîne de création d'un modèle 3D consiste à convertir le nuage de points en une surface, par exemple représentée sous la forme d'un maillage polygonal sur lequel on plaque des textures. L'objectif de la reconstruction de surface est de produire un modèle continu respectant autant que possible les propriétés géométriques et topologiques de la surface de l'objet numérisé. Il est important de choisir correctement le nombre de polygones en fonction de la résolution souhaitée.

31 À partir des problématiques précédemment énoncées, 64 groupes d'images (chunks) différents et indépendants ont été créés. La mise en place de repères sur les modèles, au prix d'une attention particulière attachée à la précision de leur position dans chacune des géométries, a permis d'assembler chaque partie pour arriver à construire un modèle final complet (185 145816 triangles). En comparant les résultats des modèles réalisés et les observations des points 3D, on a constaté la présence d'erreurs résiduelles d'environ $5 \mathrm{~cm}$ sur des séquences de 500 images. En fait, cette valeur varie 
aussi en fonction de la qualité de la surface étudiée. Dans les zones les mieux conservées et les plus riches en détails, le nombre de points caractéristiques détectés est plus élevé, leur qualité est meilleure et les résidus sont plus petits. Lorsqu'on détecte des zones couvertes de sable, les points caractéristiques sont moins nombreux et de moindre qualité.

L'utilisation des repères sur des points spécifiques, dont l'espacement est connu, est la technique normalement utilisée pour la mise à l'échelle des modèles. Dans le cas spécifique du Danton, l'on ne disposait pas de mires positionnées sur ou autour de l'épave. La mise à l'échelle du modèle 3D a cependant été rendue possible grâce aux plans de construction du cuirassé que les recherches en archives avaient livrés. Cette façon non conventionnelle d'opérer est bien sûr sujette à discussion au regard du niveau de précision que l'on ambitionne habituellement mais dans le cadre spécifique $\mathrm{du}$ «Projet Danton» cet aspect habituellement important ne présentait pas d'inconvénient majeur et l'on a en conséquence accepté la légère imprécision induite.

\section{Le modèle photogrammétrique du navire : une contribution majeure à l'analyse du site}

33 La chaîne de traitement 3D permet de générer des données comportant des millions de points et des maillages qui sont manipulables sur des machines relativement «standard » mais particulièrement lourdes à mettre en œuvre. En premier lieu, la taille très importante des données d'entrée est un facteur qui complique la construction de modèles de surface, parfois excessivement du fait de la redondance des données ou d'un niveau de détail trop élevé par rapport aux applications mobilisées ${ }^{20}$. Souvent, il est important de pouvoir modifier aisément la forme des modèles, pour les corriger, pour réduire la complexité d'un modèle maillé en appliquant des algorithmes de simplification de maillage, ou dans le cadre de l'application d'algorithmes de compression pour réduire le coût de stockage des modèles. Une autre question importante concerne alors le choix d'une représentation adaptée aux traitements et aux opérations d'édition à effectuer.

Dans le cas du « Projet Danton ", la réalisation d'un modèle tridimensionnel à très haute définition a permis d'analyser les conditions de conservation de l'épave un siècle après son naufrage. Une réflexion sur la structure architecturale du bâtiment a mis facilement en évidence les zones les plus atteintes au cours du combat au point d'occasionner son naufrage rapide. Les sections du cuirassé d'emblée foudroyées par l'attaque du U-64 se situent sur l'avant bâbord de l'épave. Elles se signalent par la présence de tôles déchirées et broyées. La zone arrière du blockhaus est également densément détruite et n'a été reconstruite pour le modèle qu'avec une grande difficulté en raison de l'état des vestiges. La disparition de certains éléments architecturaux, tels les cheminées, les mâts et les tourelles de 305, pourrait n'être en revanche que la conséquence du naufrage. S'il est difficile de définir les mouvements du navire après qu'il a chaviré, la découverte d'une section de poupe littéralement arrachée et déportée à près de $25 \mathrm{~m}$ sur le flanc bâbord montre que l'arrière du bâtiment a frappé le sol en premier avec une grande brutalité [fig. 13]. La violence de l'événement est au demeurant visible sur les images sonar fournies par la société Galsi ${ }^{21}$ : sur une grande superficie alentour de l'épave, on observe à la surface du sol des séries de « ridains », ou 
petites dunes, peut-être formés par l'onde de choc qui a potentiellement parcouru le terrain à la manière des ondes générées par un tremblement de terre.

La haute définition du modèle 3D a, enfin, permis de mettre en évidence ou de confirmer la localisation des principales zones où le processus de corrosion s'est mis à l'œuvre.

Figure 13

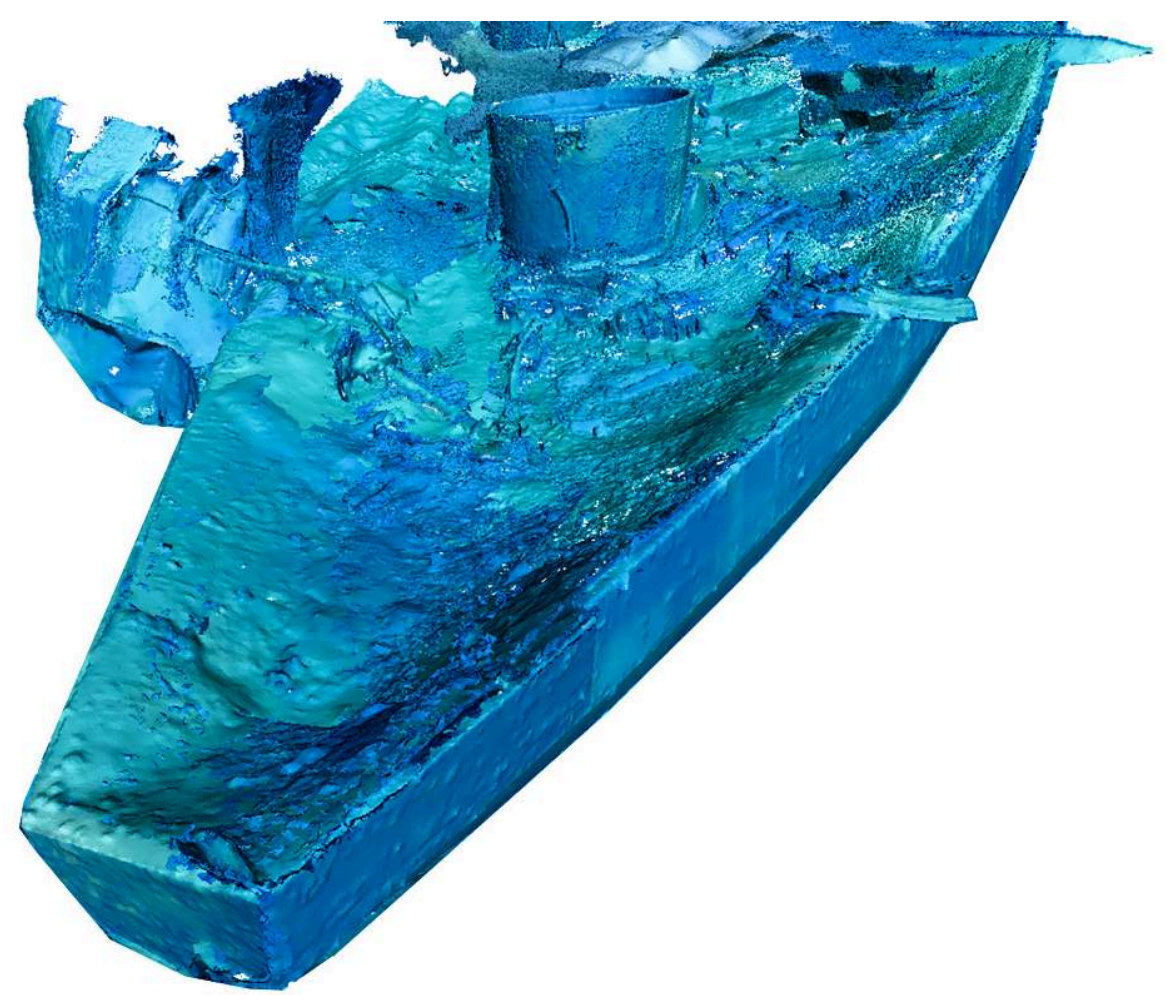

Modélisation 3D de la partie arrière du navire couverte de sédiment.

Traitement : Daniela Peloso (Ipso Facto) / cliché : Drassm, ministère de la Culture.

\section{Restituer au public sa mémoire engloutie}

Aujourd'hui, grâce au travail des archéologues et à la reconstruction photogrammétrique réalisée sur le Danton, l'épave, en dépit de sa profondeur, a retrouvé sa place dans le patrimoine sensible des grands conflits contemporains. Accessible hier aux seuls chercheurs, elle est désormais à la portée du public qui devrait bientôt visiter le Danton, à la Cité de la mer de Cherbourg (Manche) et au musée national de la Marine de Paris, comme s'ils étaient eux-mêmes en plongée sur le site. Ils pourront ce faisant découvrir "en direct " l'importance et l'intérêt du patrimoine immergé de la Première Guerre mondiale. Couplé avec des outils d'information géographique, le relevé 3D pourrait offrir à l'avenir un système de visualisation dynamique et interactif par géoréférencement de l'ensemble des données informatives et descriptives collectées ${ }^{22}$. Le modèle photogrammétrique 3D a par ailleurs été exploité dans un système de visualisation virtuelle qui s'interface avec des données archéologiques grâce aux applications spécifiques basées sur les concepts SIG. Le site a fourni à cet égard un ensemble important de données 3D incluant des références 
spatiales et des descriptions morphologiques de tous les artefacts observés. À partir d'une vue détaillée du Danton, où seraient visibles son armement, ses équipements et tous les composants du bateau, on pourrait désormais enrichir aisément le modèle 3D d'informations et de documentation iconographique [fig. 14].

Figure 14

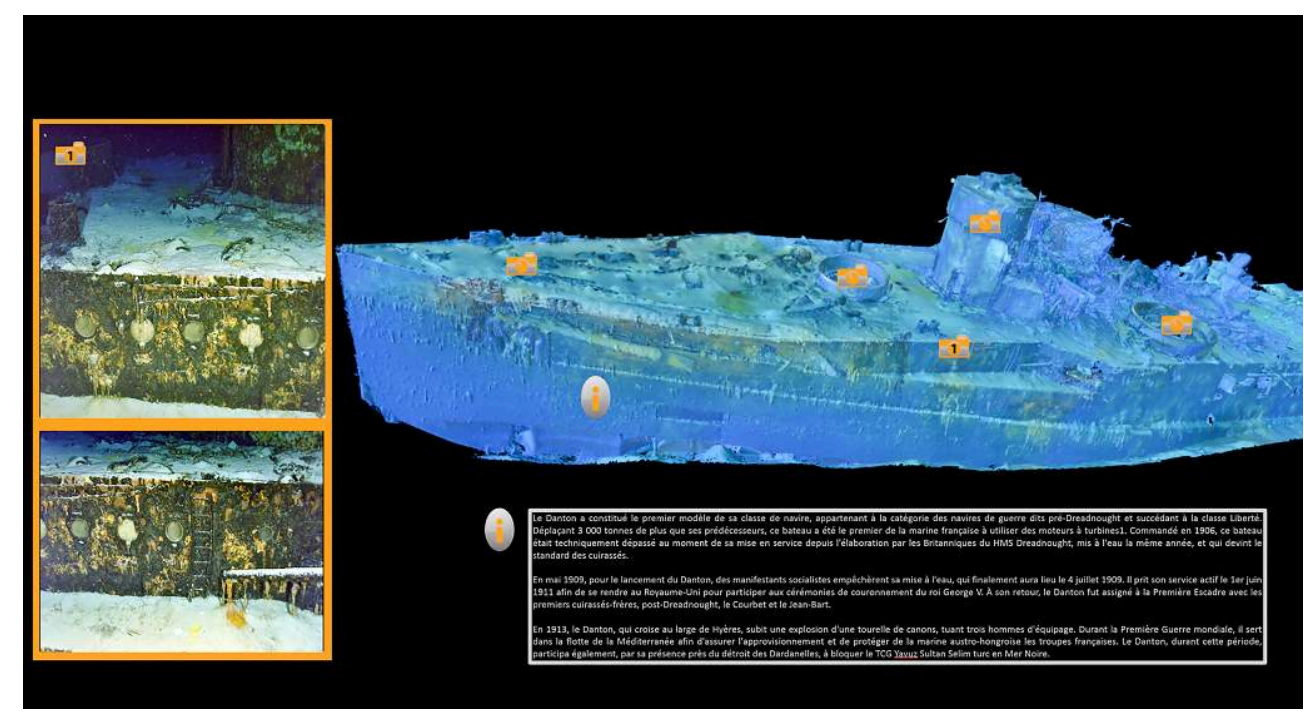

Le modèle 3D enrichi d'informations et de documentation iconographique.

Traitement : Daniela Peloso (Ipso Facto) / photos : Frédéric Osada (Images Explorations) / cliché : Drassm, ministère de la Culture.

Ce relevé 3D pourrait aussi servir de base à la fabrication de fac-similés virtuels ou physiques, ou comme support de produits culturels, éducatifs ou touristiques destinés à l'ensemble des publics ${ }^{23}$. On a ainsi d'ores et déjà simulé, depuis un ROV, une visite virtuelle de l'épave posée au fond de la Méditerranée. Cette itinérance ludique offre à tout un chacun un moyen intéressant d'explorer, cent ans après son naufrage, une épave qui gît par plusieurs centaines de mètres de fond, hors de portée des plongeurs et des submersibles de loisir. Cette expérience éducative permet de visualiser dans son ensemble un «monstre » d'acier qu'aucune plongée ne permettrait jamais, par suite de la taille de l'épave et de l'obscurité qui y règne, de visionner autrement que par courte section [fig. 15]. Pour donner au public un accès immédiat à l'épave, on a enfin aussi envisagé de produire des impressions 3D de maquettes de l'épave. 
Figure 15

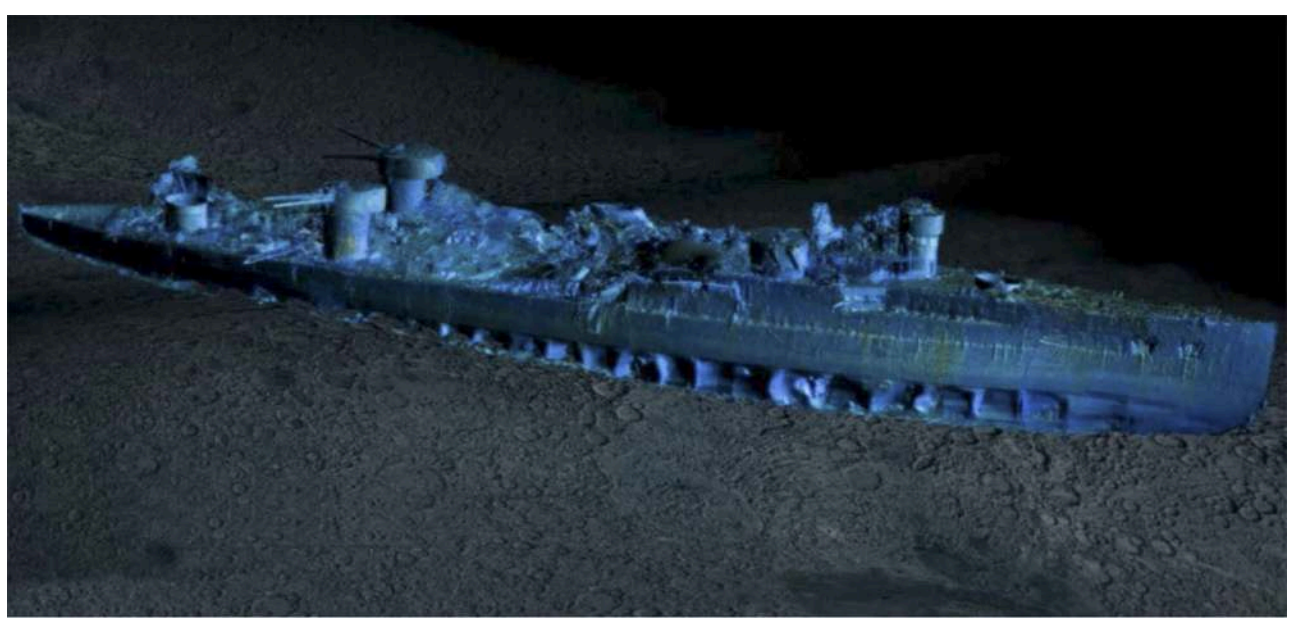

Capture d'écran d'une visualisation animée du modèle 3D du Danton.

Traitement : Daniela Peloso (Ipso Facto) / cliché : Drassm, ministère de la Culture.

Coproduit par le Drassm et la société de production de documentaires scientifiques Gédéon Programmes, un film de 26 minutes tourné pendant les opérations de 2015 et 2016, Le Mystère du cuirassé Danton, a été présenté en avant-première à l'Unesco, l'un des partenaires du projet ${ }^{24}$, le 30 mai 2017. Il a ensuite été diffusé à plusieurs reprises sur les chaînes Science \& Vie TV et RMC Découverte ${ }^{25}$.

Pour l'archéologue, l'enjeu est désormais de disposer très vite d'un fac-similé qui lui permette de finaliser une série d'observations et de vérifications à peine amorcées au cours de l'opération menée in situ. Il lui garantira aussi de pouvoir préserver des informations que la corrosion et les bactéries devraient malheureusement faire disparaitre dans un proche avenir.

\section{Le monde change : enquête en eaux profondes}

$\mathrm{Au}$ bilan, l'équipement conçu et construit pour les besoins de la mission de modélisation de l'épave du Danton s'est montré à la hauteur des espérances qu'on avait placées dans cette innovation. Des marges d'amélioration, certes, demeurent, en particulier au regard des hublots des caissons photo puisque ces derniers ne permettent pas d'avoir une image nette sur la totalité du champ. Mais, pour l'heure, ils ont tout de même permis de réaliser en sept jours et deux campagnes la couverture 3D exhaustive du Danton puis d'en conduire à bien la photogrammétrie.

41 La voie des abysses et des épaves perdues par très grand fond est ainsi aujourd'hui ouverte. Les mêmes archéologues qui ont œuvré sur le Danton, ou d'autres, s'y sont d'ailleurs prestement engouffrés. Depuis 2016, les spécialistes du Drassm ont mobilisé la même logistique pour réaliser la planimétrie de sept épaves antiques localisées par très grande profondeur au large de la Corse orientale.

Ainsi, un demi-siècle après la création du Drassm, un nouvel épisode de l'histoire de l'archéologie sous-marine, celui des enquêtes en eaux profondes, est à l'évidence sur le point de s'écrire. Le "Projet Danton " en aura été l'inspirateur et le préambule scientifique. On ne peut assurément que s'en réjouir ! 


\section{NOTES}

1. Adopté le 24 juillet 1905 par le ministre de la Marine Gaston Thomson, le programme de construction amorcé en 1906 comportait la construction de six bâtiments de 18300 tonnes. Outre le Danton, cette série, dite "classe Danton », comportait les cuirassés Mirabeau, Voltaire, Condorcet, Diderot et Vergniaud. Confiée au port de Brest, la construction du Danton obligea les autorités de l'arsenal à consentir à de gros efforts d'investissement dans les infrastructures et l'outillage afin de faire face à la réalisation d'un navire de si fort tonnage.

2. Lors de son lancement, le 22 mai 1909, en présence d'une foule de notables et de dames apprêtées, le navire a parcouru 47 mètres sur la rampe avant de s'arrêter sans qu'on ne puisse plus le mouvoir. La pente était insuffisante et le poids unitaire trop élevé. Ce n'est donc que le 4 juillet suivant que la carène d'acier toucha l'eau pour la première fois. On n'avait pas jugé opportun cette fois d'élever une tribune et d'y convier des invités...

3. Le Danton était doté d'une cuirasse de 150 à $260 \mathrm{~mm}$ d'épaisseur, soit une protection d'acier de 3640 tonnes. Propulsé par des turbines à vapeur Parsons alimentées par 26 chaudières à charbon Belleville $\left(18 \mathrm{~kg} / \mathrm{m}^{2}\right)$ développant une puissance totale de $22500 \mathrm{CV}$, il était capable de se déplacer à 20 nœuds et disposait d'une autonomie de plus de 8000 milles, soit près de $15000 \mathrm{~km}$.

4. Né le 31 janvier 1862 à Rochefort, Joseph Paul Marcel Delage est entré dans la Marine en 1878. Aspirant le 5 octobre 1881, enseigne de vaisseau le 5 octobre 1883, lieutenant de vaisseau le 16 novembre 1888, capitaine de frégate le 3 mars 1904, capitaine de vaisseau le 13 février 1911, il fut nommé commandant du Danton le 24 décembre 1915. Il avait auparavant servi ou commandé à la mer sur le cuirassé Montcalm, l'aviso Vigilant, le croiseur Milan, les sous-marins Gymnote et Gustave-Zédé, le transport Isère et les cuirassés Charles-Martel, Henri-IV et Bruix. Comme commandant du $1^{\mathrm{er}}$ régiment de fusiliers marins, il s'était également illustré en octobre 1914 lors des batailles de l'Yser et de Dixmude où il avait été blessé.

5. Né le 7 septembre 1884 à Sonderborg, ville danoise alors allemande, Robert Moraht a intégré la marine impériale en avril 1901 comme Seekadett. Kapitänleutnant le 6 décembre 1911, il prend le commandement du U-Boot U-64 le 15 avril 1916. Capturé par les Anglais le 17 juin 1918, il termine la Première Guerre mondiale en captivité au camp de Colsterdale, dans le Yorkshire, avant d'être nommé Korvetten Kapitän le 5 février 1920 et d'accéder à la retraite le 31 juillet de la même année. Réintégré dans la Kriegsmarine en 1940, nommé Fregatten Kapitän le 4 avril 1942, il occupe de 1942 à 1945 diverses responsabilités comme capitaine de port en Norvège à Kirkenes, Aalesund et Bergen puis sur l'île de Bornholm avant d'être fait prisonnier par les Russes en mai 1945. Détenu jusqu'en octobre 1948, il s'éteint le 26 août 1956 à Hambourg. Avec 45 navires coulés et 3 navires endommagés, il compte au nombre des commandants de U-Boot les plus «titrés » de la Première Guerre mondiale. En 1933, il a publié chez Schlegel une autobiographie sous le titre Werwolf der Meere. "U-64 » jagt den Feind (« Le loup-garou des mers. U-64 à la poursuite de l'ennemi »). Il y raconte sa vie sur le $U-64$ et le torpillage du Danton.

6. Les archives du Service historique de la Défense conservent un dossier fourni, notamment photographique, sur le Danton (SHD. Département Marine, SHM 7 DDI 782). On doit aux capitaines de vaisseau Serge Thébaut et Dominique Brisou l'essentiel de nos connaissances sur les singularités techniques du Danton. Le Drassm s'est pour sa part préoccupé d'assurer le récolement de toutes les archives postérieures au naufrage (rapport d'enquête, procédures d'indemnisation, etc.). Ce dossier très nourri a été réuni à notre demande par Jean-Luc Lahitte (COREDO). Que ces trois chercheurs trouvent ici l'expression de nos plus chaleureux remerciements.

7. Halomonas titanicae est une bactérie isolée par les chercheurs canadiens sur l'épave du Titanic. Par grand fond, la quantité d'oxygène est huit à dix fois inférieure à celle de la surface mais des 
centaines de colonies vivantes se développent, dont des archées et des bactéries. C'est en règle générale la famille des «bactéries sulfato-réductrices" qui est la cause d'une corrosion irrémédiable des aciers et autres fers puddlés... Dans cette famille, plusieurs dizaines d'espèces cohabitent. Leurs processus vitaux ont pour conséquence une libération acide qui « ronge » le métal sous-jacent.

8. DRAP Pierre, MERAD Djamal, MAHIDDINE Amine, SEINTURIER Julien, PELOSO Daniela, BOI Jean-Marc, CHEMISKY Bertrand \& LONG Luc, «Underwater photogrammetry for archaeology. What will be the next step? ", International Journal of heritage in the digital era, vol. 2, 2013, p. 375394.

9. On citera notamment le Black Sea Maritime Archaeology Project piloté par l'université de Southampton (Royaume-Uni) et la réalisation d'une modélisation 3D du Titanic par la société américaine Ocean Gate.

10. LUDVIGSEN Martin, SORTLAND Bjørn \& SINGH Hanumant, «Applications of geo-referenced underwater photo mosaics in marine biology and archaeology ", Oceanography, 2007, vol. 20, $\mathrm{n}^{\circ} 4$, p. 140-149.

11. Apte à intervenir jusqu'à $1500 \mathrm{~m}$ de fond, le Perseo est un ROV «standard " produit par la société Ageotech. Capable d'emporter une charge utile de $40 \mathrm{~kg}$, cette machine a été louée pour l'occasion à la société marseillaise Copetech-SM. Ses dimensions sont de 130 x 95 x $70 \mathrm{~cm}$ et son poids est de $170 \mathrm{~kg}$.

12. La perfection optique n'a pas été atteinte sur toute l'étendue de l'ouverture angulaire de ce premier hublot du caisson photographique. Ceci s'est traduit par un temps d'acquisition majoré dû à la nécessité d'augmenter le taux de recouvrement entre images voisines. De plus, cela a imposé de recourir à des « masques » pour ignorer les bords des images lors de la reconstruction photogrammétrique, comme expliqué dans la suite de l'article. Pour cette raison, le hublot devra faire l'objet d'une étude complémentaire et être corrigé dans la perspective de futures missions de reconstruction 3D.

13. Le GAPS est produit par la société française iXblue.

14. Inertial Measurement Unit.

15. Élaboration photogrammétrique et modélisation 3D réalisées par Daniela Peloso (Ipso Facto).

16. SCHETTINI Raimond \& CORCHS Silvia, «Underwater image processing. State of art of restoration and image enhancement methods ", EURASIP journal on advances in signal processing, vol. 2010, 2010, p. 1-14.

17. GIJSENIJ Arjan, GEVERS Theo \& WEIJER, Joost van de, «Computational color constancy. Survey and experiments ", IEEE transactions on image processing, vol. 20, n 9, 2011, p. 2475-2489.

18. BALLETTI Caterina, BELTRAME Carlo, COSTA, Elisa GUERRA, Francesco \& VERNIER Paolo, "Underwater photogrammetry and 3D reconstruction of marble cargos shipwreck», The International Archives of the photogrammetry, remote sensing and spatial information sciences, vol. XL-5/ W5, 2015, p. 7-13.

19. L'élaboration photogrammétrique a été réalisée avec le logiciel Agisoft Photoscan.

20. ALLÈGRE Rémi, CHAINE Raphaëlle \& AKKOUCHE Samir, «Reconstruction dynamique de surface par convection géométrique", Revue des sciences et technologies de l'information. Série «Technique et science informatiques", vol. 26, n 8, "Informatique graphique », 2007, pp. 1029-1056.

21. http://www.galsi.it/fr/faqs/societe

22. DRAP Pierre et al., « Underwater photogrammetry end object modeling. A case study of Xlendi wreck in Malta ", Sensors, vol. 15, n 12, 2015, p. 30351-30384 [en ligne], https://www.mdpi.com/ 1424-8220/15/12/29802/htm [lien valide en juin 2020].

23. VERGNIEUX Robert \& GILIGNY François, «Pour un usage raisonné de la 3D en archéologie », Les Nouvelles de l'archéologie, $\mathrm{n}^{\circ}$ 146, «La 3D en archéologie», 2016 [en ligne], http:// journals.openedition.org/nda/3818 [lien valide en juin 2020]. 
24. Dès l'origine, l'Unesco a été associée au Projet Danton, ainsi que l'Académie de marine. Il s'agissait dans les deux cas de sensibiliser le public à la nécessaire protection et à la valorisation du patrimoine immergé de la Première Guerre mondiale. Parvenus à cent ans d'âge, les vestiges engloutis du conflit 1914-1918 sont désormais placés sous la protection de la Convention Unesco de 2001 sur la Protection du patrimoine culturel subaquatique que la France a ratifiée en février 2013.

25. La restitution photogrammétrique du Danton a également fait l'objet d'un long reportage présenté dans le journal télévisé de 20 heures de la chaîne TF1, le 16 décembre 2017. Sa diffusion a bénéficié de 7,6 millions de téléspectateurs, soit la seconde audience record de l'année 2017.

\section{RÉSUMÉS}

De 1914 à 1918, les marines militaires, de commerce et de pêche ont joué un rôle de premier plan et payé un lourd tribut par suite notamment du développement de la guerre sous-marine. Curieusement, les vestiges sous-marins hérités de ce conflit ont été très longtemps négligés cependant que les hommes disparus en mer n'étaient eux-mêmes que rarement évoqués. Conscients de cette lacune, les archéologues sous-marins français ont décidé en 2013 de mettre en œuvre un ambitieux projet afin que le public puisse mieux appréhender cette page engloutie de notre histoire maritime. Ainsi est né le « Projet Danton ».

Torpillé le 19 mars 1917 par le sous-marin allemand U-64, le cuirassé français Danton, construit en 1909, a coulé en moins d'une heure, emportant au cœur de ses 19000 tonnes d'acier près de 300 marins. Largement oubliée, son épave a presque miraculeusement été retrouvée lorsqu'un robot a localisé en janvier 2008, par 1025 mètres de fond au large de la Sardaigne une vaste anomalie que des études ont ensuite identifiée comme le cuirassé disparu.

Deux missions in situ ont été effectuées en 2015 et 2016 par le Département français des recherches archéologiques subaquatiques et sous-marines (Drassm). Ces opérations ont eu recours à un robot doté d'équipements spécifiquement conçus pour filmer et photographier à très grande profondeur. En moins de six jours, les archéologues ont pu ainsi acquérir plus de 42000 photographies haute définition et 27 vidéos des vestiges. Ce travail a donné lieu à une modélisation 3D de l'épave qui permettra bientôt au public de visiter le Danton comme s'il était lui-même en plongée sur le site.

Le «Projet Danton » a en outre conduit à développer une technologie particulièrement novatrice qui permet d'envisager désormais de réaliser très rapidement la couverture 3D d'épaves situées jusqu'à $2000 \mathrm{~m}$ de profondeur.

From 1914 to 1918, military, merchant and fishing fleets played a leading role... but paid a heavy price when submarines took the war beneath the waves. Curiously enough, underwater remains dating from this terrible conflict were for a long time ignored, while the men lost at sea were themselves rarely mentioned. In 2013, French underwater archaeologists, aware of this omission, decided to undertake an ambitious project to raise awareness among the public of this sunken page of our maritime history. And the result was 'Danton Project'.

Torpedoed on 19 March 1917 by the German submarine U-64, the French battleship Danton, built in 1909, sank in less than an hour, taking close to 300 sailors along with her 19,000 tons of steel. Largely forgotten, the Danton was discovered quite by chance in January 2008 when an 
underwater robot located, at a depth of 1,025 metres off Sardinia, a huge anomaly that subsequent surveys identified as the wreck of the lost battleship.

Two surveys were carried out in situ in 2015 and 2016 by France's Department of Underwater and Underwater Archaeological Research.

These operations involved the deployment of a robot fitted with specially designed equipment for filming and photographing at great depths. In less than six days the archaeologists were able to acquire more than 42,000 high-definition photographs and twenty-seven videos of the remains. This work laid the foundation for a 3D computer model of the wreck which will soon be made available to the public so they can visit the Danton as if they were actually diving to the site. The 'Danton Project' also led to a particularly innovative technological development which promises to dramatically speed up the 3D mapping of wrecks lying at depths of down to 2,000 metres.

\section{INDEX}

Mots-clés : patrimoine culturel submergé, archéologie sous-marine, Danton, cuirassé français, ROV, restitution photogrammétrique, 3D

Keywords : underwater cultural heritage, underwater archaeology, Danton, French battleship, ROV, 3D computer model

\section{AUTEURS}

\section{MICHEL L'HOUR}

Conservateur général du Patrimoine, directeur du Département des recherches archéologiques subaquatiques et sous-marines (Drassm, ministère de la Culture, Marseille, France), membre de l'Académie de Marine, membre du conseil scientifique et technique (STAB) de l'Unesco

(Convention de 2001).

michel.lhour@culture.gouv.fr

\section{DANIELA PELOSO}

Docteure en sciences et technologies, archéologue, responsable du pôle Technologie 3D du bureau d'étude et de recherche en océanographie et en archéologie subaquatique et sous-marine Ipso Facto (Marseille, France).

daniela.peloso@ipsofacto.coop

\section{FRANCA CIBECCHINI}

Adjointe au directeur pour l'archéologie préventive et programmée, responsable du littoral corse, Département des recherches archéologiques subaquatiques et sous-marines (Drassm, ministère de la Culture, Marseille, France), membre du Centre Camille-Jullian (UMR CNRS 7299). franca.cibecchini@culture.gouv.fr

\section{DENIS DEGEZ}

Ingénieur d'étude, archéologue sous-marin au département des Recherches archéologiques subaquatiques et sous-marines (Drassm, ministère de la Culture, Marseille, France). denis.degez@culture.gouv.fr 


\section{VINCENT CREUZE}

Laboratoire d'informatique, de robotique et de microélectronique de Montpellier (LIRMM, Univ Montpellier, CNRS, Montpellier, France).

vincent.creuze@lirmm.fr

FRÉDÉRIC OSADA

Images Explorations (Chalon-sur-Saône, France)

frederic.osada@wanadoo.fr

\section{CHRISTOPHE LECLERCQ}

Société Posseïdon LDY (Monaco)

contact@l-d-y.com 